\title{
Effects of first- and second-generation tyrosine kinase inhibitor therapy on glucose and lipid metabolism in chronic myeloid leukemia patients: a real clinical problem?
}

\author{
Alessandra Iurlo ${ }^{1,2}$, Emanuela Orsi ${ }^{3}$, Daniele Cattaneo ${ }^{1}$, Veronica Resi ${ }^{3}$, Cristina \\ Bucelli ${ }^{1}$, Nicola Orofino ${ }^{1}$, Mariarita Sciumè ${ }^{1}$, Chiara Elena ${ }^{4}$, Valeria Grancini ${ }^{3}$, Dario \\ Consonni ${ }^{5}$, Ester Maria Orlandi ${ }^{4}$ and Agostino Cortelezzi ${ }^{1}$ \\ ${ }^{1}$ Oncohematology Division, IRCCS Ca' Granda - Maggiore Policlinico Hospital Foundation, and University of Milan, Milan, \\ Italy \\ ${ }^{2}$ Oncohematology Unit of the Elderly, IRCCS Ca' Granda - Maggiore Policlinico Hospital Foundation, and University of Milan, \\ Milan, Italy \\ 3 Endocrinology and Diabetes Unit, Department of Medical Sciences, IRCCS Ca' Granda - Maggiore Policlinico Hospital \\ Foundation, and University of Milan, Milan, Italy \\ ${ }^{4}$ Oncology-Hematology Department, Hematology Unit, Fondazione IRCCS Policlinico San Matteo, Pavia, Italy \\ ${ }^{5}$ Epidemiology Unit, Department of Preventive Medicine, IRCCS Ca' Granda - Maggiore Policlinico Hospital Foundation, \\ Milan, Italy \\ Correspondence to: Alessandra lurlo, email: aiurlo@policlinico.mi.it \\ Keywords: chronic myeloid leukemia, imatinib, dasatinib, nilotinib, diabetes mellitus, metabolic syndrome \\ Received: June 18,2015 Accepted: August 21, $2015 \quad$ Published: September 10, 2015
}

This is an open-access article distributed under the terms of the Creative Commons Attribution License, which permits unrestricted use, distribution, and reproduction in any medium, provided the original author and source are credited.

\section{ABSTRACT}

Background: Tyrosine kinase inhibitors (TKIs) have dramatically changed the prognosis of patients with chronic myeloid leukemia (CML). They have a distinct toxicity profile that includes glycometabolic alterations: i.e. diabetes mellitus (DM), impaired fasting glucose (IFG), and the metabolic syndrome (MS). The aim of this study was to evaluate the prevalence of these alterations in a cohort of CML-chronic phase patients treated with imatinib, dasatinib or nilotinib.

Methods: The study involved 168 consecutive CML-chronic phase patients with no history of DM/IFG or MS. Anthropometric and metabolic parameters were assessed, and DM/IFG and MS were diagnosed based on the criteria of the American Diabetes Association and the National Cholesterol Education Program-Adult Treatment Panel III, respectively.

Results: The nilotinib group had significantly higher levels of fasting plasma glucose, insulin, C-peptide, insulin resistance, and total and LDL cholesterol than the imatinib and dasatinib groups. DM/IFG were identified in $25 \%$ of the imatiniband dasatinib-treated patients, and $33 \%$ of those in the nilotinib cohort $(p=0.39$ vs imatinib and $p=0.69$ vs dasatinib). A diagnosis of MS was made in $42.4 \%$ of the imatinib-treated patients, $37.5 \%$ of the dasatinib-treated patients, and $36.1 \%$ of the nilotinib-treated patients ( $p=0.46$ vs imatinib and $p=0.34$ vs dasatinib).

Conclusions: Treatment with nilotinib does not seem to induce DM/IFG or the MS to a significantly higher extent than imatinib or dasatinib, though it causes a worse glycometabolic profile. These findings suggest the need for a close monitoring of glucose and lipid metabolism and a multidisciplinary approach in patients treated with nilotinib. 


\section{INTRODUCTION}

Until 1999, drug therapy for chronic myeloid leukemia (CML) $[1,2]$ was limited to non-specific agents such as busulfan, hydroxyurea, cytosine arabinoside, and interferon- $\alpha($ IFN- $\alpha)$ [3]. The development of small-molecule tyrosine kinase inhibitors (TKIs) has dramatically changed patients' prognosis as they potently interfere with the interaction between the $B C R-A B L 1$ protein and adenosine triphosphate (ATP), and block the proliferation of the malignant clone [4]. This "targeted" approach has significantly changed the natural history of CML and improved 10-year overall survival from less than $20 \%$ to $80-90 \%$ [2,5]. Imatinib mesylate was the first TKI to be approved by the US Food and Drug Administration for the treatment of patients with CML-chronic phase, followed in 2007 by the second-generation TKIs dasatinib and nilotinib [6-11].

TKIs approved for first- and second-line treatment of CML-chronic phase have a distinct toxicity profile that includes glycometabolic alterations such as diabetes mellitus (DM), impaired fasting glucose (IFG), and the metabolic syndrome (MS), a cluster of metabolic abnormalities characterized by insulin resistance [12].
Based on its age distribution, it can be predicted that prevalence of CML will increase with the increasing age of the general population, and that this will lead to a significantly higher risk of developing these metabolic disorders upon treatment with certain TKIs. In fact, a large phase III trial comparing the efficacy of nilotinib and imatinib showed that hyperglycemia occurred in $50 \%$ of patients treated with nilotinib $300 \mathrm{mg}$ b.i.d., $53 \%$ of those treated with nilotinib $400 \mathrm{mg}$ b.i.d., and only $31 \%$ of those treated with imatinib $400 \mathrm{mg} /$ day; yet, none of these patients discontinued TKI therapy because of hyperglycemia or had serious diabetes-related adverse events [13]. However, no data are available concerning the prevalence of DM/IFG and the MS in "real-life", unselected CML patients on TKI therapy.

Purposes of this study were (a) to assess the prevalence of glycometabolic alterations (DM/IFG, MS) in a cohort of CML-chronic phase patients on TKI therapy; and (b) to identify which parameter(s) should be evaluated at diagnosis and during treatment to help clinicians to choose the most appropriate TKI for each patient from a metabolic point of view.

Table 1: Clinical and laboratory features of $168 \mathrm{CML}-\mathrm{chronic}$ phase patients treated with imatinib, dasatinib or nilotinib.

\begin{tabular}{|c|c|c|c|c|}
\hline & $\begin{array}{c}\text { Imatinib group } \\
\text { (n. 92) }\end{array}$ & $\begin{array}{c}\text { Dasatinib group } \\
(\mathrm{n}, 40)\end{array}$ & $\begin{array}{c}\text { Nilotinib group } \\
(\mathrm{n} .36)\end{array}$ & P-value* \\
\hline Age (years), median (range) & $59.2(21.2-87.5)$ & $54.9(24.5-82.6)$ & $46.0(25.1-79.0)$ & 0.0044 \\
\hline Male/Female, $n$ & $49 / 43$ & $23 / 17$ & $20 / 16$ & 0.90 \\
\hline $\begin{array}{l}\text { Length of treatment (months), median } \\
\text { (range) }\end{array}$ & $93.0(0.4-165.5)$ & $41.6(3.0-97.1)$ & $16.1(0.1-70.0)$ & 0.0001 \\
\hline $\mathrm{BMI}\left(\mathrm{Kg} / \mathrm{m}^{2}\right)$, mean $(\mathrm{SD})$ & $25.9(4.8)$ & $24.8(3.7)$ & $26.3(3.5)$ & 0.24 \\
\hline FPG $(\mathrm{mg} / \mathrm{dl})$, mean $(\mathrm{SD})$ & $98.0(22.8)$ & $92.8(12.0)$ & $104.7(18.9)$ & 0.0069 \\
\hline $\mathrm{HbA}_{1 c}(\%)$, mean $(\mathrm{SD})$ & $5.5(0.7)$ & $5.5(0.9)$ & $5.6(0.6)$ & 0.54 \\
\hline Insulin $(\mu \mathrm{UI} / \mathrm{ml})$, median (range) & $7.9(2.3-33.1)$ & $6.7(2.7-29.8)$ & $11.0(6.2-29.5)$ & 0.0001 \\
\hline C-peptide $(\mu \mathrm{mol} / \mathrm{L})$, median (range) & $2.3(1.1-8.5)$ & $1.9(0.9-4.2)$ & $2.8(1.8-4.8)$ & 0.0003 \\
\hline HOMA-IR, median (range) & $1.7(0.6-12.0)$ & $1.4(0.6-7.9)$ & $2.8(1.4-7.0)$ & 0.0001 \\
\hline HOMA-\%B, median (range) & $94.3(22.8-550.8)$ & $92.3(42.1-364.1)$ & $99.7(34.9-393.2)$ & 0.26 \\
\hline Total cholesterol (mg/dl), median (range) & $177.0(119.0-265.0)$ & $189.0(114.0-337.0)$ & $217.5(140.0-297.0)$ & 0.0001 \\
\hline HDL cholesterol (mg/dl), median (range) & $48.0(20.0-98.0)$ & $50.0(23.0-109.0)$ & $53.0(32.0-87.0)$ & 0.09 \\
\hline Triglycerides (mg/dl), median (range) & $102.0(32.0-506.0)$ & $111.0(46.0-457.0)$ & $104.0(36.0-249.0)$ & 0.59 \\
\hline LDL cholesterol (mg/dl), mean (SD) & $105.2(32.1)$ & $124.2(35.4)$ & $140.0(36.5)$ & 0.0001 \\
\hline SBP (mmHg), median (range) & $130.0(100.0-180.0)$ & $122.5(100.0-170.0)$ & $125.0(110.0-150.0)$ & 0.28 \\
\hline DBP $(\mathrm{mmHg})$, mean $(\mathrm{SD})$ & $79.0(7.8)$ & $77.9(8.8)$ & $80.1(6.5)$ & 0.39 \\
\hline Waist circumference $(\mathrm{cm})$, mean $(\mathrm{SD})$ & $94.3(15.7)$ & $90.4(12.8)$ & $94.2(12.2)$ & 0.40 \\
\hline $\mathrm{DM} / \mathrm{IFG}, \mathrm{n}(\%)$ & $23(25.0)$ & $10(25.0)$ & $12(33.3)$ & 0.61 \\
\hline MS, n (\%) & $39(42.4)$ & $15(37.5)$ & $13(36.1)$ & 0.77 \\
\hline
\end{tabular}

$\mathrm{CML}=$ chronic myeloid leukemia; $\mathrm{BMI}=$ body mass index $; \mathrm{FPG}=$ fasting plasma glucose $\mathrm{SD}=$ standard deviation; $\mathrm{HbA} 1 \mathrm{c}=$ hemoglobin A1c; HOMA-IR= Homeostasis Model Assessment - Insulin Resistance; HOMA-\%B= Homeostasis Model Assessment $-\beta$-cell function; $\mathrm{SBP}=$ systolic blood pressure; $\mathrm{DBP}=$ diastolic blood pressure; $\mathrm{DM}=$ diabetes mellitus; $\mathrm{IFG}=$ impaired fasting glucose; $\mathrm{MS}=$ metabolic syndrome.

*From Kruskal-Wallis (continuous variables) or chi-squared (categorical variables) test. 
Table 2: Multiple regression analysis adjusted for centre, gender, age, BMI, and length of treatment, comparing imatinib versus nilotinib group.

\begin{tabular}{|c|c|c|c|}
\hline & \multirow{2}{*}{ Imatinib group (n.92) } & \multicolumn{2}{|c|}{ Nilotinib group (n. 36) } \\
\cline { 3 - 4 } & & Coefficient (95\% CI)* & P-value* \\
\hline FPG (mg/dl) & Reference & $9.8(1.9 ; 17.8)$ & 0.015 \\
\hline Insulin (\%) & Reference & $17.4(6.2 ; 30.3)$ & 0.002 \\
\hline C-peptide (\%) & Reference & $11.6(4.1 ; 19.7)$ & 0.002 \\
\hline HOMA-IR (\%) & Reference & $22.1(9.4 ; 37.7)$ & $<0.001$ \\
\hline HOMA-\%B (\%) & Reference & $2.0(-9.5 ; 10.5)$ & 0.76 \\
\hline Total cholesterol (\%) & Reference & $11.6(7.3 ; 16.2)$ & $<0.001$ \\
\hline HDL cholesterol (\%) & Reference & $6.2(1.0 ; 11.6)$ & 0.02 \\
\hline Triglycerides (\%) & Reference & $3.6(-5.8 ; 13.9)$ & 0.48 \\
\hline LDL cholesterol (mg/dl) & Reference & $42.4(27.0 ; 57.8)$ & $<0.001$ \\
\hline & & OR (95\% CI)** & P-value \\
\hline DM/IFG & Reference & $1.7(0.5 ; 5.8)$ & 0.39 \\
\hline MS & Reference & $0.6(0.2 ; 2.4)$ & 0.46 \\
\hline
\end{tabular}

$\mathrm{BMI}=$ body mass index; $\mathrm{CI}=$ confidence interval; $\mathrm{OR}=$ odds ratio; $\mathrm{FPG}=$ fasting plasma glucose; HOMA-IR= Homeostasis Model Assessment - Insulin Resistance; HOMA- $\%$ B= Homeostasis Model Assessment - $\beta$-cell function; $\mathrm{DM}=$ diabetes mellitus; $\mathrm{IFG}=$ impaired fasting glucose; $\mathrm{MS}=$ metabolic syndrome.

*From multiple linear regression models.

**From multiple logistic regression models.

\section{RESULTS}

One hundred and sixty-eight consecutive patients diagnosed as having CML-chronic phase and treated with imatinib $(n=92)$, dasatinib $(n=40)$ or nilotinib $(n$ $=36$ ) entered the study. Among them, 107 were in firstline, 53 in second-line, and the remaining 8 in third-line treatment. Moreover, only 3 patients changed TKI because of intolerance to the previous treatment, whereas all the other patients were resistant. Our cohort included 92 males $(54.8 \%)$, and their median age at the time of recruitment was 56.0 years (range 21.2-87.5) (Table 1).

In detail, at the time of assessment, patients in the imatinib group were significantly older and were receiving treatment for longer than those in the other groups. There were significant differences in fasting plasma glucose (FPG), insulin, C-peptide, and the Homeostasis Model Assessment - Insulin Resistance (HOMAIR) index, but not in body mass index (BMI), waist circumference, hemoglobin $(\mathrm{Hb}) \mathrm{A}_{1 \mathrm{c}}$ and HOMA - $\beta$-cell function (HOMA-\%B). Moreover, there were significant differences in total and LDL cholesterol, but not in HDL cholesterol, triglycerides, and systolic and diastolic blood pressure.
Table 2, 3, and 4 show the results of the multiple regression analyses adjusted for centre, gender, age, BMI and length of treatment, and expressed as absolute differences or percent changes. FPG, insulin, C-peptide, and HOMA-IR were all significantly higher in the nilotinib group than in the other two groups, whereas there was no difference in HOMA-\%B. Total, HDL, and LDL cholesterol levels did not differ significantly between the dasatinib and nilotinib groups, but they were significantly higher in both groups than in the imatinib group, except for HDL cholesterol between dasatinib- and imatinibtreated individuals. No difference in triglyceride levels was found among the three groups.

On the basis of clinical and laboratory data, 45 patients $(26.8 \%)$ were classified as having type $2 \mathrm{DM} /$ IFG and $67(41.4 \%)$ as having the MS, with no significant between-treatment group differences at both univariate (Table 1) and multiple regression analyses (Table 2, 3 and 4$)$. Only 2 patients (1.2\%) discontinued TKI therapy because of the development of glycometabolic alterations, whereas 3 subjects (1.8\%) required a specific treatment for hyperglycemia, without discontinuing TKI therapy. 
Table 3: Multiple regression analysis adjusted for centre, gender, age, BMI, and length of treatment, comparing dasatinib versus nilotinib group.

\begin{tabular}{|c|c|c|c|}
\hline & \multirow{2}{*}{ Dasatinib group (n. 40) } & \multicolumn{2}{|c|}{ Nilotinib group (n. 36) } \\
\cline { 3 - 4 } & Coefficient (95\% CI)* & P-value* \\
\hline FPG (mg/dl) & Reference & $9.7(2.5 ; 16.9)$ & 0.009 \\
\hline Insulin (\%) & Reference & $22.1(11.6 ; 33.6)$ & $<0.001$ \\
\hline C-peptide (\%) & Reference & $16.2(9.4 ; 24.0)$ & $<0.001$ \\
\hline HOMA-IR (\%) & Reference & $27.1(16.2 ; 40.5)$ & $<0.001$ \\
\hline HOMA-\%B (\%) & Reference & $7.3(-4.9 ; 20.9)$ & 0.27 \\
\hline Total cholesterol (\%) & Reference & $3.9(-0.8 ; 8.3)$ & 0.10 \\
\hline HDL cholesterol (\%) & Reference & $3.0(-2.0 ; 8.3)$ & 0.24 \\
\hline Triglycerides (\%) & Reference & $-5.8(-14.8 ; 4.1)$ & 0.23 \\
\hline LDL cholesterol (mg/dl) & Reference & $14.8(-2.5 ; 32.1)$ & 0.09 \\
\hline & & OR (95\% CI)*** & P-value** \\
\hline DM/IFG & Reference & $1.3(0.4 ; 4.1)$ & 0.69 \\
\hline MS & Reference & $0.5(0.2 ; 1.9)$ & 0.34 \\
\hline
\end{tabular}

$\mathrm{BMI}=$ body mass index; $\mathrm{CI}=$ confidence interval; $\mathrm{OR}=$ odds ratio; $\mathrm{FPG}=$ fasting plasma glucose; HOMA-IR = Homeostasis Model Assessment - Insulin Resistance; HOMA-\%B= Homeostasis Model Assessment $-\beta$-cell function; $\mathrm{DM}=$ diabetes mellitus; $\mathrm{IFG}=$ impaired fasting glucose; $\mathrm{MS}=$ metabolic syndrome.

*From multiple linear regression models.

**From multiple logistic regression models.

\section{DISCUSSION}

Each of the TKIs approved for treatment of patients with CML-chronic phase has a distinct toxicity profile that should be carefully considered when prescribing TKI treatment. It is now well known that first- (imatinib) and second- (dasatinib, nilotinib) generation TKIs act on different molecular targets [14], and that their side effects are mainly due to the inhibition not only of $B C R-A B L 1$ but also of other tyrosine kinases such as c-kit, PDGFR, $S r c$ or $E P H B 4$. Imatinib mainly causes peripheral edema, whereas dasatinib predisposes some patients to pleural effusions and, to a lesser extent, pulmonary arterial hypertension [15], and inhibits platelet function [16,17]. Both drugs do not negatively impact on glucose metabolism and may even improve FPG levels and allow a reduction of the dosage of anti-hyperglycemic drugs [18-21]. The positive effect of imatinib might be due to an improvement of both insulin secretion, via inhibition of $\beta$-cell apoptosis [22] due to increased activation of antiapoptotic transcription factor $N F-k B$ or decreased activation of the proapoptotic $M A P K J N K$, and insulin sensitivity in peripheral tissues, via inhibition of tumour necrosis factor- $\alpha$ production [23] and/or endoplasmic reticulum stress [24,25]. Conversely, nilotinib was shown to induce hyperglycemia in a subset of non-diabetic CML patients [13,26,27]. The underlying mechanism is controversial as a previous analysis of 10 patients demonstrated a reduction in insulin sensitivity [28], whereas a case report showed a reversible decrease in insulin secretion [29] under nilotinib therapy. Regarding lipid profile, total, HDL, and LDL cholesterol levels were found to increase significantly in patients treated with nilotinib, and to decrease in those receiving imatinib [30]. The latter effect might be due to the fact that imatinib can act also on $P D G F R$, which may induce phosphorylation of LDL receptor-related protein [31].

Our cross-sectional analysis assessed the prevalence of DM/IFG and the MS in a large series of "real-life", unselected CML-chronic phase patients during TKI treatment and estimated both insulin sensitivity and $\beta$-cell function in these individuals. We found a nonsignificant trend toward an increase in the prevalence of DM/IFG, but not the MS, in CML-chronic phase patients treated with nilotinib $v s$ those receiving imatinib or dasatinib. Moreover, individuals on nilotinib showed significantly higher FPG, insulin, C-peptide, and total and LDL cholesterol levels and HOMA-IR values, with no differences in $\mathrm{HbA}_{1 \mathrm{c}}$ and HOMA-\%B. 
Table 4: Multiple regression analysis adjusted for centre, gender, age, BMI, and length of treatment, comparing imatinib versus dasatinib group.

\begin{tabular}{|c|c|c|c|}
\hline & \multirow{2}{*}{ Imatinib group (n. 92) } & \multicolumn{2}{|c|}{ Dasatinib group (n. 40) } \\
\cline { 3 - 4 } & Reference & $0.2(-4.9 ; 5.2)$ & 0.95 \\
\hline FPG (mg/dl) & Reference & $-3.9(-12.2 ; 5.7)$ & 0.42 \\
\hline Insulin (\%) & Reference & $-4.4(-10.4 ; 2.0)$ & 0.18 \\
\hline C-peptide (\%) & Reference & $-3.9(-13.1 ; 7.3)$ & 0.48 \\
\hline HOMA-IR (\%) & Reference & $-4.9(-13.9 ; 6.2)$ & 0.37 \\
\hline HOMA-\%B (\%) & Reference & $7.8(4.1 ; 11.6)$ & $<0.001$ \\
\hline Total cholesterol (\%) & Reference & $3.0(-2.0 ; 8.3)$ & 0.22 \\
\hline HDL cholesterol (\%) & Reference & $9.4(0.2 ; 20.9)$ & 0.046 \\
\hline Triglycerides (\%) & Reference & $27.6(14.7 ; 40.5)$ & $<0.001$ \\
\hline LDL cholesterol (mg/dl) & & OR (95\% CI)** & P-value** \\
\hline & Reference & $1.3(0.4 ; 4.2)$ & 0.60 \\
\hline DM/IFG & Reference & $1.1(0.4 ; 3.5)$ & 0.85 \\
\hline MS & &
\end{tabular}

$\mathrm{BMI}=$ body mass index $\mathrm{CI}=$ confidence interval; $\mathrm{OR}=$ odds ratio; $\mathrm{FPG}=$ fasting plasma glucose; HOMA-IR= Homeostasis Model Assessment - Insulin Resistance; HOMA-\%B= Homeostasis Model Assessment $-\beta$-cell function; $\mathrm{DM}=$ diabetes mellitus; $\mathrm{IFG}=$ impaired fasting glucose; $\mathrm{MS}=$ metabolic syndrome.

*From multiple linear regression models.

**From multiple logistic regression models.

The increase in FPG, but not in $\mathrm{HbA}_{1 \mathrm{c}}$ levels, in patients treated with nilotinib suggests that the hyperglycemic effect of this drug may not be clinically meaningful and that it likely does not affect post-prandial glucose concentrations. This interpretation is consistent with the higher HOMA-IR, but not HOMA-\%B values, indicating an impairment of insulin sensitivity with no effect on $\beta$-cell function. Also the lack of significant increases in the prevalence of DM/IFG observed in patients receiving nilotinib, which is at variance with a previous report from a phase III trial [13], seems to argue against the concept that the effect of this drug on glucose metabolism represents a real clinical problem. This view is consistent with a previous report that fasting hyperglycemia does not progress to type $2 \mathrm{DM}$ in nilotinib-treated individuals [27]. However, our prevalence data might have been influenced by a selection bias caused by the significant inter-groups differences in age and duration of TKI treatment, which may have not been accounted for by statistical adjustment. In fact, patients on nilotinib were younger and with a shorter treatment duration than those on imatinib and dasatinib. Taken together, our data, while excluding a potent diabetogenic effect of nilotinib, do support the need for a close monitoring of glucose (and lipid) metabolism in patients receiving this drug and for a multidisciplinary approach to manage rises of FPG (and cholesterol) levels during treatment. In clinical practice, it would be appropriate to measure FPG, cholesterol, and possibly $\mathrm{HbA}_{1 \mathrm{c}}$ levels before treatment to identify subjects at risk to develop glycometabolic abnormalities and to guide the choice of the TKI. Moreover, the same parameters should be assessed at 3 months of treatment, and in the nilotinib group every 6-12 months thereafter. This strategy would be adequate for reducing cardiovascular burden associated with hyperglycemia and dyslipidemia, which may increase the risk of atherosclerosis with peripheral artery occlusion occurring with nilotinib treatment $[30,32,33]$.

Strengths of our study include the "real-life" setting and the size of the cohort, which is much larger than in previous reports [18-21,26]. Limitations include the cross-sectional design of the study, the already mentioned differences among treatment groups in terms of age and duration of TKI therapy, and the criteria for excluding the presence of DM and the MS at baseline. In addition, the fact that an oral glucose tolerance test was not performed in the study subjects might have resulted in an underestimation of the prevalence of DM.

In conclusion, this cross-sectional analysis of CMLchronic phase patients on treatment with three TKIs showed that prevalence of DM/IFG or the MS was not significantly different, though glycometabolic profile was worse in subjects treated with nilotinib than in those on imatinib or dasatinib. These findings indicate the need 
for a close monitoring of glucose and lipid metabolism and a multidisciplinary approach in patients treated with nilotinib.

\section{MATERIALS AND METHODS}

\section{Patients}

One hundred and sixty-eight consecutive CMLchronic phase patients attending the Oncohematology Division of the IRCCS Ca' Granda - Maggiore Policlinico Hospital Foundation, Milan, and the Hematology Unit of the IRCCS Policlinico San Matteo Hospital Foundation, Pavia, were recruited between May 2013 and July 2014. The study was approved by the local Ethics Committees, and all patients gave their written informed consent in accordance with the Italian guidelines for the protection of human subjects. Inclusion criteria were age $>18$ years, CML-chronic phase diagnosis based on World Health Organization 2008 criteria, and current treatment with imatinib, dasatinib or nilotinib [14]. CML patients who received only IFN- $\alpha$ therapy were excluded from the analysis, as were those with a previous history of DM/ IFG, MS, or statin treatment. FPG levels and body weights before starting TKI treatment were also available in all cases.

\section{Methods}

Anthropometric and metabolic parameters of each patient were assessed at enrollment. All patients underwent physical examination, with assessment of BMI, waist circumference, and blood pressure, and biochemical testing, including $\mathrm{HbA}_{1 \mathrm{c}}, \mathrm{FPG}$, insulin, and $\mathrm{C}$-peptide levels, and serum lipid profile.

As a surrogate measure of insulin secretion, the HOMA-\%B was calculated from fasting insulin and glucose concentrations by the formula: $\left(20 \mathrm{xIns}_{0}\right) /\left(\right.$ Gluc $_{0}-$ 3.5). The HOMA-IR index was estimated using the formulas proposed by Levy et al. [34,35].

Diabetes mellitus, IFG, and the MS were diagnosed according to the criteria of the American Diabetes Association, updated in 2010 (based on FPG and $\mathrm{HbA}_{1 \mathrm{c}}$ levels) [36], and those of the National Cholesterol Education Program - Adult Treatment Panel III [12].

\section{Statistical analysis}

The Kruskal-Wallis and chi-squared tests were used to compare continuous and categorical variables, respectively, in the treatment groups. The laboratory results were evaluated by fitting multiple linear regression models adjusted for centre, gender, age, BMI, and length of treatment. Two sets of comparisons were made: 1) nilotinib and dasatinib vs imatinib; and 2) nilotinib vs dasatinib. In the case of FPG and LDL cholesterol, the regression coefficients represent absolute differences (in $\mathrm{mg} / \mathrm{dL})$. Conversely, as the other laboratory data were right skewed, we fitted regression models using their $\log _{10}$-transformed values as dependent variables, and then calculated their percent changes using the formula: \% change $=\left[\operatorname{antilog}_{10}(\right.$ Coefficient $\left.)-1\right] \times 100$ [37]. For DM/ IFG and the MS, we used logistic regression models to calculate adjusted odds ratios (ORs) and 95\% confidence intervals (CIs), once again using the imatinib or dasatinib groups as reference [38]. The statistical analyses were made using Stata 13 software [39].

\section{ACKNOWLEDGMENTS}

This work was supported in part by grant from Fondazione Alberto e Antonietta Matarelli and AIL Associazione Italiana contro le Leucemie, Linfomi e Mieloma - Milano e Provincia ONLUS.

\section{FUNDING}

This work was supported by funds from the authors' institutions.

\section{CONFLICTS OF INTERESTS}

AI received a speaker funding from Bristol Myers Squibb, NOVARTIS, ARIAD, Pfizer and SHIRE. EO has been a member of advisory boards or a speaker with Eli Lilly, Boehringer Ingelheim, AstraZeneca, Roche, MerckSharp \& Dome and Lifescan J\&J. EMO received a speaker funding from Bristol Myers Squibb, NOVARTIS and Roche. DCa, VR, CB, NO, MS, CE, VG, DCo and AC declare that they have no conflict of interest.

\section{Author contributions}

AI was responsible for the integrity of the work as a whole

$\mathrm{AI}$ and EO designed the study

AI, EO, DCa, VR and EMO interpreted the data and wrote the manuscript

DCo made statistical analysis

AI, EMO, DCa, CB, NO, MS, CE and VG followed the patients and collected the data

AC reviewed the data and approved the manuscript

\section{REFERENCES}

1. Rowley JD. Letter: a new consistent chromosomal abnormality in chronic myelogenous leukaemia identified by quinacrine fluorescence and Giemsa staining. Nature. 
1973; 243: 290-293.

2. Jemal A, Siegel R, Xu J, Ward E. Cancer statistics, 2010. CA Cancer J Clin. 2010; 60: 277-300.

3. Silver RT, Woolf SH, Hehlmann R, Appelbaum FR, Anderson J, Bennett C, Goldman JM, Guilhot F, Kantarjian HM, Lichtin AE, Talpaz M, Tura S. An evidence-based analysis of the effect of busulfan, hydroxyurea, interferon, and allogeneic bone marrow transplantation in treating the chronic phase of chronic myeloid leukemia: developed for the American Society of Hematology. Blood. 1999; 94: $1517-1536$.

4. Okuda K, Matulonis U, Salgia R. Factor independence of human myeloid leukemia cell lines is associated with increased phosphorylation of the proto-oncogene Raf-1. Exp Hematol. 1994; 22: 1111-1117.

5. Raitano AB, Halpern JR, Hambuch TM. The Bcr-Abl leukemia oncogene activates Jun kinase and requires Jun for transformation. Proc Natl Acad Sci USA. 1995; 92: 11746-11750.

6. Druker BJ, Lydon NB. Lessons learned from the development of an abl tyrosine kinase inhibitor for chronic myelogenous leukemia. J Clin Invest. 2000; 105: 3-7.

7. Lombardo LJ, Lee FY, Chen P, Norris D, Barrish JC, Behnia K, Castaneda S, Cornelius LA, Das J, Doweyko AM, Fairchild C, Hunt JT, Inigo I, et al. Discovery of N-(2-chloro-6-methyl-phenyl)22-(6-(4-(2-hydroxyethyl)piperazin-1-yl)22-methylpyrimidin-4-ylamino)thiazole5-carboxamide (BMS-354825), a dual Src/Abl kinase inhibitor with potent antitumor activity in preclinical assays. J Med Chem. 2004; 47: 6658-6661.

8. O'Hare T, Walters DK, Stoffregen EP, Jia T, Manley PW, Mestan J, Cowan-Jacob SW, Lee FY, Heinrich MC, Deininger MW, Druker BJ. In vitro activity of Bcr-Abl inhibitors AMN107 and BMS-354825 against clinically relevant imatinib resistant $\mathrm{Abl}$ kinase domain mutants. Cancer Res. 2005; 65: 4500-4505.

9. Tokarski JS, Newitt JA, Chang CY, Cheng JD, Wittekind M, Kiefer SE, Kish K, Lee FY, Borzillerri R, Lombardo LJ, Xie D, Zhang Y, Klei HE. The structure of Dasatinib (BMS-354825) bound to activated ABL kinase domain elucidates its inhibitory activity against imatinib resistant ABL mutants. Cancer Res. 2006; 66: 5790-5797.

10. Shah NP, Tran C, Lee FY, Chen P, Norris D, Sawyers CL. Overriding imatinib resistance with a novel ABL kinase inhibitor. Science. 2004; 305: 399-401.

11. Weisberg E, Manley PW, Breitenstein W, Brüggen J, Cowan-Jacob SW, Ray A, Huntly B, Fabbro D, Fendrich G, Hall-Meyers E, Kung AL, Mestan J, Daley GQ, et al. Characterization of AMN107, a selective inhibitor of native and mutant Bcr-Abl. Cancer Cell. 2005; 7: 129-141.

12. National Cholesterol Education Program (NCEP) Expert Panel on Detection, Evaluation, and Treatment of High Blood Cholesterol in Adults (Adult Treatment Panel III). Third report of the National Cholesterol Education Program
(NCEP) expert panel on detection, evaluation, and treatment of high blood cholesterol in adults (Adult Treatment Panel III) final report. Circulation. 2002; 106: 3143-3421.

13. Larson RA, Kim DW, Issaragrilsil S, le Coutre P, Dorlhiac Llacer PE, Etienne G, Clark RE, Flinn I, Nakamae H, Hochhaus A, Saglio G, Kantarjian HM, Donohue B, et al. Efficacy and Safety of Nilotinib (NIL) vs Imatinib (IM) in Patients (pts) With Newly Diagnosed Chronic Myeloid Leukemia in Chronic Phase (CML-CP): Long-Term Follow-Up (f/u) of ENESTnd. Blood. 2014; 124: abstract 4541.

14. Baccarani M, Deininger MW, Rosti G, Hochhaus A, Soverini S, Apperley JF, Cervantes F, Clark RE, Cortes JE, Guilhot F, Hjorth-Hansen H, Hughes TP, Kantarjian HM, et al. European LeukemiaNet recommendations for the management of chronic myeloid leukemia: 2013. Blood. 2013; 122: 872-884.

15. Montani D, Bergot E, Günther S, Savale L, Bergeron A, Bourdin A, Bouvaist H, Canuet M, Pison C, Macro M, Poubeau P, Girerd B, Natali D, et al. Pulmonary arterial hypertension in patients treated by dasatinib. Circulation. 2012; 125: 2128-2137.

16. Quintas-Cardama A, Han X, Kantarjian H, Cortes J. Tyrosine kinase inhibitor-induced platelet dysfunction in patients with chronic myeloid leukemia. Blood. 2009; 114: 261-263.

17. Quintás-Cardama A, Kantarjian H, Ravandi F, O'Brien S, Thomas D, Vidal-Senmache G, Wierda W, Kornblau $\mathrm{S}$, Cortes J. Bleeding diathesis in patients with chronic myeloid leukemia receiving dasatinib therapy. Cancer. 2009; 115: 2482-2490.

18. Breccia M, Muscaritoli M, Aversa Z, Mandelli F, Alimena G. Imatinib mesylate may improve fasting blood glucose in diabetic $\mathrm{Ph}+$ chronic myelogenous leukemia patients responsive to treatment. J Clin Oncol. 2004; 22: 4653-4655.

19. Veneri D, Franchini M, Bonora E. Imatinib and regression of Type 2 Diabetes. New Engl J Med. 2005; 352: 10491050.

20. Breccia M, Muscaritoli M, Cannella L, Stefanizzi C, Frustaci A, Alimena G. Fasting glucose improvement under dasatinib treatment in an accelerated phase chronic myeloid leukemia patient unresponsive to imatinib and nilotinib. Leuk Res. 2008; 32: 1626-1628.

21. Ono K, Suzushima H, Watanabe Y, Kikukawa Y, Shimomura T, Furukawa N, Kawaguchi T, Araki E. Rapid amelioration of hyperglycaemia facilitated by dasatinib in a chronic myeloid leukemia patient with type 2 diabetes mellitus. Intern Med. 2012; 51: 2763-2766.

22. Hägerkvist R1, Makeeva N, Elliman S, Welsh N. Imatinib mesylate (Gleevec) protects against streptozotocin-induced diabetes and islet cell death in vitro. Cell Biol Int. 2006; 30 : 1013-1017.

23. Wolf AM, Wolf D, Rumpold H, Ludwiczek S, Enrich B, Gastl G, Weiss G, Tilg H. The kinase inhibitor imatinib 
mesylate inhibits TNF-alfa production in vitro and prevents TNF-dependent acute hepatic inflammation. Proc Natl Acad Sci U S A. 2005; 102: 13622-13627.

24. Han MS, Chung KW, Cheon HG, Rhee SD, Yoon $\mathrm{CH}$, Lee MK, Kim KW, Lee MS. Imatinib mesylate reduces endoplasmic reticulum stress and induces remission of diabetes in db/db mice. Diabetes. 2009; 58: 329-336.

25. Louvet C, Szot GL, Lang J, Lee MR, Martinier N, Bollag G, Zhu S, Weiss A, Bluestone JA. Tyrosine kinase inhibitors reverse type 1 diabetes in nonobese diabetic mice. Proc Natl Acad Sci USA. 2008; 105: 18895-18900.

26. Breccia M, Muscaritoli M, Gentilini F, Latagliata R, Carmosino I, Rossi Fanelli F, Alimena G. Impaired fasting glucose level as metabolic side effect of nilotinib in nondiabetic chronic myeloid leukemia patients resistant to imatinib. Leuk Res. 2007; 31: 1770-1772.

27. Breccia M, Loglisci G, Salaroli A, Serrao A, Alimena G. Nilotinib-mediated increase in fasting glucose level is reversible, does not convert to type 2 diabetes and is likely correlated with increased body mass index. Leuk Res. 2012; 36: e66-67.

28. Racil Z, Razga F, Drapalova J, Buresova L, Zackova D, Palackova M, Semerad L, Malaskova L, Haluzik M, Mayer J. Mechanism of impaired glucose metabolism during nilotinib therapy in patients with chronic myelogenous leukemia. Haematologica. 2013; 98: 124-126.

29. Ito Y, Miyamoto T, Chong Y, Maki T, Akashi K, Kamimura T. Nilotinib exacerbates diabetes mellitus by decreasing secretion of endogenous insulin. Int J Hematol. 2013; 97: 135-138.

30. Rea D, Mirault T, Cluzeau T, Gautier JF, Guilhot F, Dombret H, Messas E. Early onset hypercholesterolemia induced by the 2nd-generation tyrosine kinase inhibitor nilotinib in patients with chronic phase-chronic myeloid leukemia. Haematologica. 2014; 99: 1197-1203.

31. Breccia M, Molica M, Alimena G. How tyrosine kinase inhibitors impair metabolism and endocrine system function: A systematic updated review. Leuk Res. 2014; 38: 1392-1398.

32. Tefferi A. Nilotinib treatment-associated accelerated atherosclerosis: when is the risk justified? Leukemia. 2013; 27: 1939-1940.

33. Valent P, Hadzijusufovic E, Schernthaner GH, Wolf D, Rea D, le Coutre P. Vascular safety issues in CML patients treated with BCR/ABL1 kinase inhibitors. Blood. 2015; 125: 901-906.

34. Levy JC, Matthews DR, Hermans MP. Correct homeostasis model assessment (HOMA) evaluation uses the computer program. Diabetes Care. 1998; 21: 2191-2192.

35. Gutt M, Davis CL, Spitzer SB, Llabre MM, Kumar M, Czarnecki EM, Schneiderman N, Skyler JS, Marks JB. Validation of the insulin sensitivity index (ISI $(0,120)$ ): comparison with other measures. Diabetes Res Clin Pract. 2000; 47: 177-184.
36. Standard of Medical Care in Diabetes-ADA, Diabetes Care, vol 33, Suppl 1, Jan 2010.

37. Armitage P, Berry G, Matthews JNS. Statistical Methods in Medical Research (Fourth Edition). Oxford: Blackwell Science Ltd; 2002.

38. Hosmer DW, Lemeshow S. Applied Logistic Regression. New York, NY: John Wiley \& Sons; 1989.

39. StataCorp. 2013. Stata: Release 13. Statistical Software. College Station, TX:StataCorp LP. 